\title{
PTEN/Akt Axis is Involved in Somatic Cell Reprogramming to Mouse iPS Cells
}

\author{
Tomoe Ueyama ${ }^{1}$, Shu Nakao $^{1}$, Tasuku Tsukamoto ${ }^{1}$, Dai Ihara ${ }^{1}$, Yukihiro Harada ${ }^{1}$, Yuka Akagi ${ }^{1}$, Sae Nakagawa $^{1}$, \\ Yasuyuki S Kida ${ }^{3}$, Takahiro Sogo ${ }^{2}$ and Teruhisa Kawamura*1
}

${ }^{1}$ Department of Biomedical Sciences, Japan

${ }^{2}$ Global Innovation Research Organization, Japan

${ }^{3}$ Research Center for Stem Cell Engineering, Japan

Received: 啙: November 28, 2018; Published: 制: December 10, 2018

*Corresponding author: Teruhisa Kawamura, Laboratory of Stem Cell and Regenerative Medicine, Department of Biomedical Sciences, Japan

\begin{abstract}
Induced pluripotent stem cells (iPSCs) are highly expected to apply for regeneration therapy and disease modeling, although the efficiency of reprogramming to iPSCs remains not high enough. We previously reported that inhibition of p53 tumor suppressor gene exhibits a remarkable increase in the efficiency of reprogramming to iPSCs. While p53 is degraded by Mdm2 and Mdmx with E3 ligase activity, phosphatase and tensin homologue (PTEN) inhibits Mdm-dependent p53 degradation, through phosphoinositol-3-kinase/Akt-dependent and -independent pathways. Therefore, we hypothesized that targeting PTEN may affect the efficiency of reprogramming to iPSCs. Using mouse embryonic fibroblasts prepared from Ptenflox/flox mice, Cre-loxP-based gene ablation of Pten revealed an increase in Akt phosphorylation and downregulation of the protein levels of p53 and one of its downstream targets, p21, resulting in efficient iPSC colony formation. In addition, CRISPR-Cas9-based knock-down of Pten gene also promoted the reprogramming efficiency. Conversely, overexpression of PTEN increased the levels of p53 and p21 and decreased the number of iPSC colonies. Moreover, co-infection of a constitutively active form of Akt (CA Akt) perturbed 4F-induced upregulation of p53 and p21, and increased iPSC production, which was canceled by overexpression of p53. We further found that negative cell cycle regulators, 19Arf and p16Ink4a were also upregulated during reprogramming, both of which were downregulated by CA Akt. Therefore, deficiency of PTEN improved the reprogramming efficiency by activation of Akt at least in part through downregulation of p19Arf and p16Ink4a as well as significant inhibition of p53/p21 pathway. Taken together, regulation of PTEN/Akt axis would be a promising strategy to enhance the efficiency of reprogramming to iPSCs.

Keywords: Induced Pluripotent Stem Cell; Reprogramming; PTEN/Akt pathway; p53/p21 pathway

Abbreviations: CA: Constitutively Active; CRISPR: Clustered Regularly Interspaced Short Palindromic Repeats, ESC: Embryonic Stem Cell; HA: Hemagglutinin; IPSC: Induced Pluripotent Stem Cell; MEF: Mouse Embryonic Fibroblast; PIP3: Phosphatidylinositol $(3,4,5)$-trisphosphate; PTEN, Phosphatase and Tensin Homolog; 4F: reprogramming four factors
\end{abstract}

\section{Introduction}

Somatic cells including embryonic or adult fibroblasts are experimentally reprogrammed to induced pluripotent stem cells (iPSCs) with transduction of the defined four factors (4F), Oct4, Sox2, Klf4, and c-Myc [1, 2]. Following the reprogramming process, somatic cells acquire the capabilities of unlimited replication and pluripotency, as observed in embryonic stem cells (ESCs). Moreover, patient-derived iPSCs are applicable to disease modeling and drug screening as well as cell therapy after gene correction by the genome engineering. Reprogramming efficiency to iPSCs, however, remains not high enough, and defined reprogramming enhancers have been under investigation. Therefore, it would be valuable to examine how somatic cells are reprogrammed to iPSCs and to determine what molecules regulate this process. As one of the clues to address these issues above, previous studies including ours demonstrated that iPSC induction efficiency is remarkably increased by inhibition of $\mathrm{p} 53 / \mathrm{p} 21$ pathway [3-7]. 
p53, a tumor suppressor gene (known as TP53 in humans), is known to be stabilized and activated in response to a variety of cellular stresses including DNA damage signaling, oncogene activation, and forced expression of reprogramming factors, Klf4 or cMyc. Following these stress signal transductions, p53 is stabilized through inactivation of an E3 ubiquitin ligases, Mdm2 [8] and Mdmx [9]. While activation of p53 induces transcription of p21 leading to cell cycle arrest and DNA repair, p53 also induces apoptosis-related genes such as Bax, Noxa, and Puma, followed by cell death [10]. In contrast, reducing the p53 signaling by modulating E3 activity of Mdm2 or Mdmx promotes not only cell growth and proliferation but also reprogramming to iPSCs $[3,11]$. This may explain, in part, the reason why reprograming efficiency is limited to the low level. For functional regulation of p53 in the course of iPSC reprogramming, in the present study, we focus on upstream factors of the p53/Mdm axis.

It is reported that activation of $\mathrm{Mdm} 2$ is regulated by phosphoinositol-3-kinase/Akt, known as a crucial regulator of cell survival, growth, differentiation, apoptosis and autophagy in various cell types [12]. When Akt is phosphorylated by phosphatidylinositol $(3,4,5)$-trisphosphate (PIP3), activated Akt promotes translocation of Mdm2 from the nucleus to the cytoplasm, thereby increased degradation of p53 protein [13-16]. It is also known that Akt activity is repressed by phosphatase and tensin homolog (PTEN), which is another well-known tumor repressor and negatively regulates PIP3 through dephosphorylation of position D3 of PIP3. In addition, it was previously reported that PTEN/Akt pathway is involved in the regulation of cell cycle, apoptosis, and tumorigenesis through the interaction with p53/p21 pathway [17]. However, involvement of the linkage between PTEN/Akt and p53 pathways in iPSC formation remains unclear. In the present study, we here examine whether modulating the signals of PTEN/Akt axis affect p53-mediated inhibition of reprogramming to iPSCs. Our data show that inhibition of PTEN or activating Akt potentially enhances the efficiency of iPSC formation.

\section{Methods}

\section{Animals}

Any experiments in this study were approved by Experimental Animal Committee of Ritsumeikan University. Animal care and experimental procedures followed the Animal Welfare Committee guidelines of Ritsumeikan University. Mice used for this study were kept in an air and humidity-conditioned room at $22-24{ }^{\circ} \mathrm{C}$ in the Animal Facility in Ritsumeikan University. Ptenflox/flox mice were purchased from Jackson Laboratory, and the homozygotes were maintained in mouse colonies. Mice were genotyped by PCR analysis of genomic DNA isolated with a standard phenol-chloroform extraction. Genotyping PCR were performed with ExTaq (Takara Bio, Japan) under standard conditions using a primer pair for knocked-in allele and wild-type allele. We used pregnant females to collect E13.5-14.5 embryos and sacrificed with dislocation of the neck prior to Cesarean section.

\section{Cell Culture and iPSC Reprogramming}

Mouse embryonic fibroblasts (MEFs) were isolated from E13.514.5 embryos of C57BL/6J or Pten flox/flox mice. These cells were cultured in MEF medium containing Dulbecco's modified eagle medium (nacalai tesque, Japan), 10\% fetal bovine serum (Nichirei Bioscience, Japan), 2 mM L-glutamine (GlutaMAX, GIBCO) and 1\% (10,000 U/L and $10 \mathrm{mM}$ ) penicillin-streptomycin (Wako, Japan). MEFs passed three to six times were used for experiments as somatic cells. Cells were cultured at $37^{\circ} \mathrm{C}, 20 \% 02$ and $5 \% \mathrm{CO} 2$ in a humidified incubator. The iPSCs were induced as previously described [3]. Briefly, retroviruses encoding Oct4, Sox2, Klf4 or cMyc were generated in HEK293T packaging cells following transfection with Lipofectamine2000 (Invitrogen). In two days after lipofection, supernatants containing viruses were collected through a $0.45-\mu \mathrm{m}$ filter. These viral vectors were infected with poly brane to MEFs. Lentiviral vectors were also produced in HEK293T cells and infected to MEFs. On day 2 of infection, cells were split and maintained under the condition for undifferentiated mouse pluripotent stem cells.

\section{Plasmid Construction and Lentivirus-Mediated Genome Editing}

The pMX vectors (pMX-mOct4, -mSox2, -mKlf4, -mc-Myc) were used for reprogramming into iPSCs as previously described [3]. pBabe-Cre was generated following modification that Cre recombinase cDNA was inserted in pBabe-Puro from Addgene. pMXhPTEN was generated by subcloning of hPTEN and was inserted into the pMXs vector with restriction enzymes, XhoI and BamHI. Referred to a previous report [18], pMX-constitutively active Akt, which is a myristylated mutant, was generated by subcloning of mAkt fused with a myristylation signal to the $\mathrm{N}$-terminus and the hemagglutinin (HA) epitope tag to the C-terminus, and was inserted into the pMXs vector with restriction enzymes, Hind III and Sal I. Lentivirus vectors for CRISPR-Cas9-based genome editing were prepared using plasmid, pLenti CRISPR (Addgene). SgRNA design and cloning were performed based on a previously reported protocol [19], with an online CRISPR design tool (CRISPR direct). The sequences of oligoes were: Sense oligonucleotides:

5'-CACCGTGTGCATATTTATTGCATCG-3' and Antisense oligonucleotides:

5'-AAACCGATGCAATAAATATGCACAC-3' for PTEN sgRNA\#1; Sense oligonucleotides:

CACCGAAACAAAAGGAGATATCAAG and Antisense oligonucleotides: AAACCTTGATATCTCCTTTTGTTTC, for PTEN sgRNA\#2. PTEN targeting sequences were cloned into BmsBIdigested pLenti CRISPR (Add gene).

\section{Immunolabeling}

To assess the reprogramming efficiency, cultured cells were immunostained at day 11-15 after transgene of $4 \mathrm{~F}$ using the VectaStain $\mathrm{ABC}$ kit and ImmPACT DAB substrate (Vector 
Laboratories) with polyclonal rabbit anti-mouse Nanog (Calbiochem) antibodies according to the manufacturer's protocol. In brief, the cells were fixed in $10 \%$ neutral phosphate-buffered formalin, permeabilized with $0.5 \%$ Triton X-100, and blocked with $5 \%$ BSA. Following overnight reaction with the primary antibodies, the cells were incubated with the biotin-conjugated anti-rabbit IgG (Vector Laboratories) for $45 \mathrm{~min}$, then reacted with streptavidinHRP complex for $30 \mathrm{~min}$. Nanog-positive colonies were visualized in DAB solution and washed in distilled water and counted the number under a stereomicroscope.

\section{Western Blotting}

Immunoblotting was performed by means of standard protocols. Protein samples were collected from cultured cells in 6 days after infection with a lysis buffer containing 4\% SDS, and sonicated and quantified with BCA method. The samples were then reduced with a 2-mercaptoethanol-containing loading buffer and boiled at $95{ }^{\circ} \mathrm{C}$ for 5 min. SDS-PAGE were performed in a $10 \%$ polyacrylamide gel. The protein ladders were transferred to a nitrocellulose membrane. The primary antibodies used were as follows: PTEN (Cell Signaling Technology, rabbit polyclonal), p16Ink4a (Santa Cruz Biotechnology, rabbit polyclonal), p19Arf (Abcam, rabbit polyclonal), p21 (Santa Cruz Biotechnology, mouse monoclonal), p53 (Cell Signaling Technology, mouse monoclonal), phospho-Akt (T308) Cell Signaling Technology, rabbit Polyclonal), Akt (Cell Signaling Technology, rabbit polyclonal), \-actin (SigmaAldrich, mouse monoclonal). Specific signals were visualized with Pierce Western Blotting substrate (Thermo Scientific) and acquired with LAS-4000 (Fuji Film).

\section{Statistical analysis}

Statistical analysis was performed using Student's t-test. All the data were expressed as mean with standard deviation, and $\mathrm{P}<0.05$ was considered significant

\section{Results}

\section{Deficiency of PTEN Promotes Efficiency of Reprogramming to iPSCs Through Activation of Akt/p53/p21 Pathway}

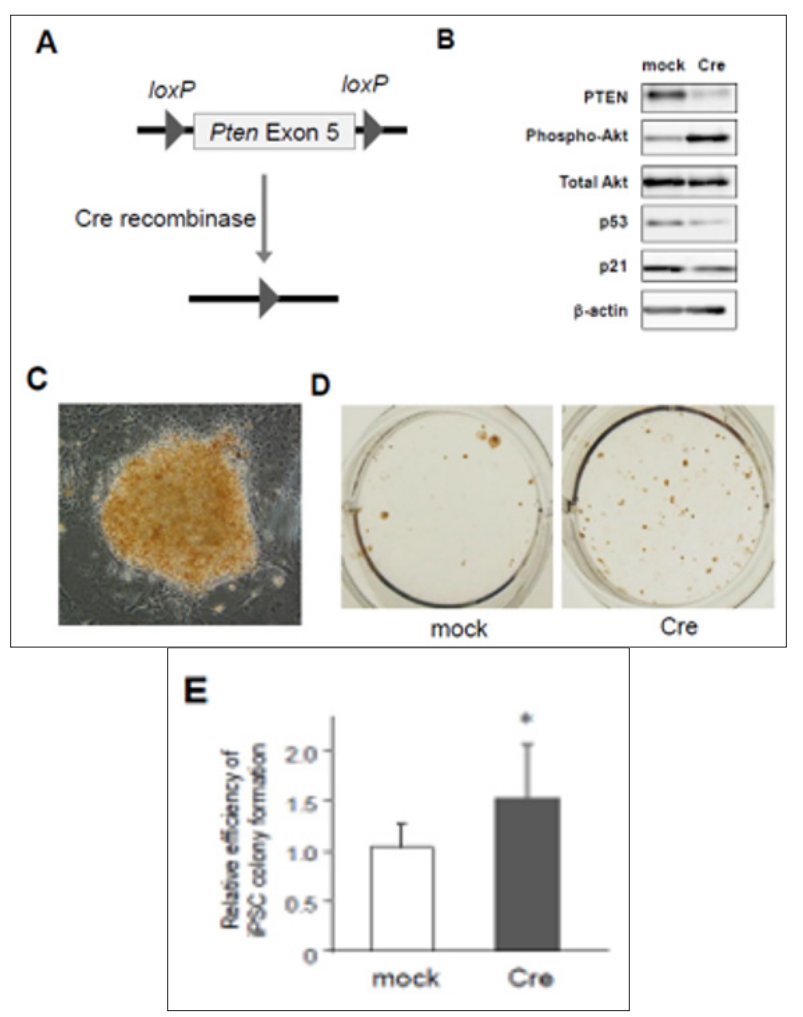

Figure 1: Activation of PTEN/Akt and p53/p21 pathways in mouse embryonic fibroblasts (MEFs). prepared from Pten conditional knockout mice

a) Scheme of Cre-loxP system to knockout Pten gene.

b) Western blotting for PTEN, phosphorylated Akt, total Akt, p53, and p21 in 4F-transduced MEFs with mock or Cre coinfection. (C) to (E) An iPSC colony induced from Pten conditional knockout MEFs

c) Photographs of an iPSC colony stained with mouse monoclonal antibodies against Nanog, an undifferentiated iPSC maker (200x).

d) Nanog-positive iPSC colonies cultured in a 12-well plate.

e) Increased number of Nanog-positive colonies in Pten knockout MEFs ( $n=6,{ }^{*} p<0.05$, error bars show s.d.). 
We first examined the signal crosstalk between PTEN/Akt and p53/p21 pathways after 4F-induced iPSC reprogramming, using a conditional knockout system. MEFs prepared from Ptenflox/flox mouse embryos were retrovirally infected with $4 \mathrm{~F}$ in addition to either mock or Cre recombinase. The exon 5 of Pten gene is flanked by the loxP sites and designed to be removed by Cre expression (Figure 1A). We then tested the effects of PTEN deficiency on the levels of phosphorylated Akt and p53 by Western blotting. Under the presence of Cre, the protein level of PTEN was almost abolished in 4F-expressing MEFs (Figure 1B). PTEN deficient cells also exhibited significant phosphorylation of Akt and downregulation of p53 and p21 (Figure 1B), indicating that PTEN dephosphorylates Akt to maintain the activation of p53/p21 pathway after 4F-infection. Since p53/p21 pathway activation is a critical barrier against iPSC formation according to previous reports including ours [3], our results above raise the possibility that PTEN may repress reprogramming efficiency.

Then, we assessed whether lack of PTEN increases the efficiency of 4F-induced iPSC colony formation. MEFs were transduced with 4F together with mock or Cre recombinase, and around ten days after infection these cells were fixed with formalin and labeled with antibodies for Nanog, one of specific markers of undifferentiated
iPSCs. Nanog-positive colonies were visualized using a standard method of immunocytochemistry (Figure 1C). The results showed that the number of Nanog-positive iPSC colonies was significantly increased in PTEN-deficient cells (Figures 1D \& 1E), in which Akt was highly phosphorylated (Figure 1B). These results suggest that deficiency of PTEN improves reprogramming efficiency to iPSCs at least through Akt-dependent pathways. Using lentivirus vectors for CRISPR/Cas9-based genome editing to disrupt the gene by frameshift mutations, we also confirmed that there was significant reduction of PTEN protein in CRISPR/Cas9-transduced cells expressing two different types of gRNAs targeting Pten gene (Figure 2A). MEFs co-infected with CRISPR/Cas9-lentivirus vector and retrovirus vectors encoding $4 \mathrm{~F}$ were cultured for iPSC formation. Immunocytochemistry revealed that significantly increased number of iPSC colonies appeared from 4F-transduced MEFs coinfected with CRISPR/Cas9 vectors expressing either gRNA \#1 or \#2 (Figure 2B). We finally performed gain-of-function experiments in MEFs overexpressing PTEN. Conversely, co-infection of Pten together with $4 \mathrm{~F}$ induced upregulation of p53 and p21 proteins during reprogramming (Figure 2C) and reduced the efficiency of reprogramming to iPSCs. Taken together, these results suggest the novel crosstalk between PTEN/Akt and p53/p21 pathways involved in reprogramming to iPSCs.

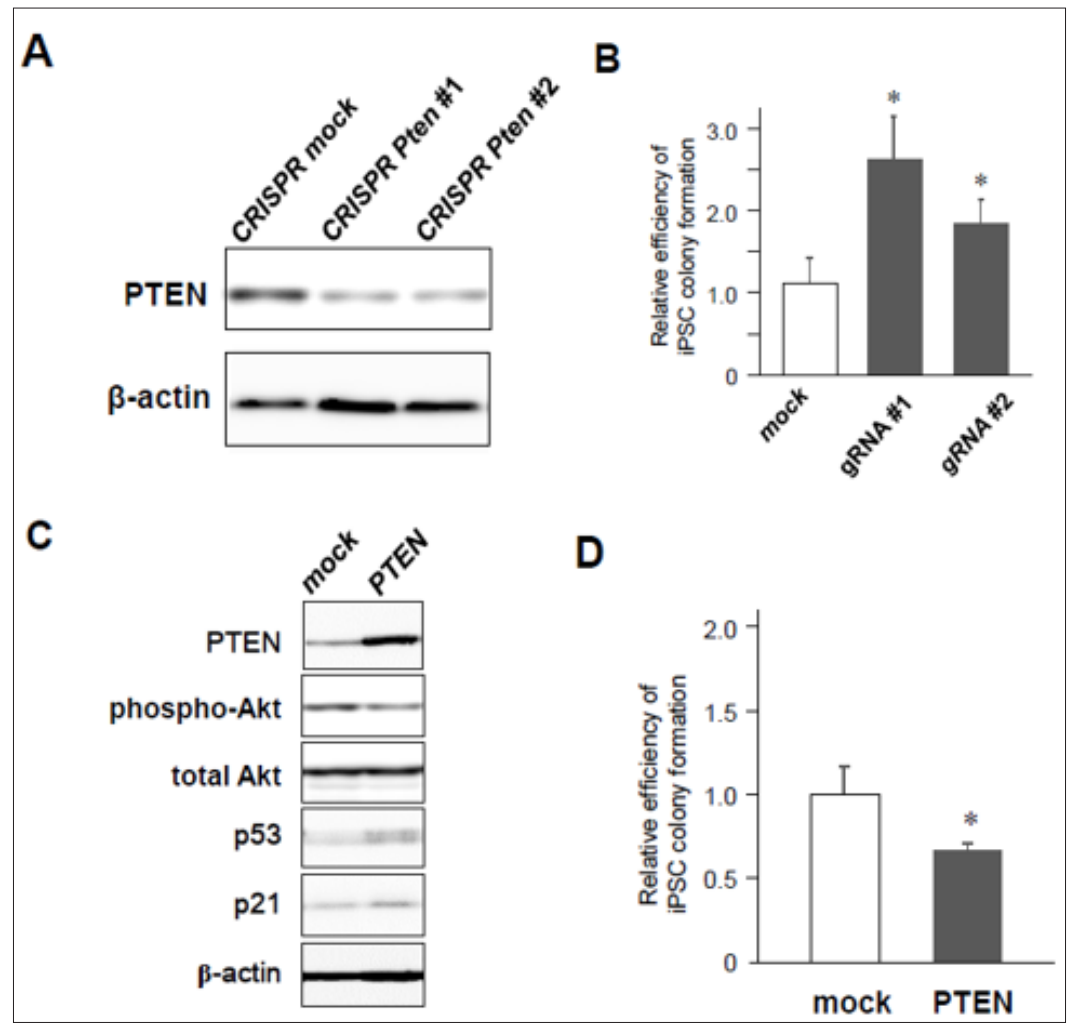

Figure 2: iPSC reprogramming efficiency from MEFs with knock-down or overexpression of PTEN.

a) Significant downregulation of PTEN in MEFs transduced with CRISPR/Cas9 against Pten gene.

b) Increased colony formation of iPSCs by CRISPR/Cas9-induced Pten knock-down ( $\mathrm{n}=3$, * $\mathrm{p}<0.05$, error bars show s.d.).

c) Western blotting of 4F-induced MEFs with mock- or PTEN-overexpression.

d) Decreased number of iPSC colony formation by co-infection of PTEN ( $n=4,{ }^{*} p<0.05$, error bars show s.d.). 


\section{Constitutively Active form of Akt Rescues Inhibition of iPSC Colony Formation}

While PTEN downregulates PIP3 and subsequently induces dephosphorylation and inactivation of Akt, our results showed that PTEN activates p53/p21 pathway leading to reduced efficiency of iPSC reprogramming. Therefore, we next examined whether activation of Akt improves reprogramming efficiency through inhibiting p53/p21 signaling. We performed reprogramming experiments using MEFs introduced with $4 \mathrm{~F}$ in addition to mock or a constitutively active form of HA-tagged Akt (CA Akt). As observed in our previous report [3], western blot analysis showed 4F-mediated upregulation of p53 and p21 (Figure 3A). Of note, coinfection of CA Akt reduced the p53/p21 protein levels (Figure 3A). Then, we performed immunolabeling with Nanog 10 to 12 days after infection and counted Nanog-positive colonies. As expected, CA Akt together with 4F significantly increased the number of iPSC colonies (Figure 3B). In contrast, this enhanced reprogramming efficiency was canceled by co-infection of p53 in reprogrammed cells induced by $4 \mathrm{~F}$ and CA Akt (Figures 3C \& 3D). These results suggest that Akt activation improves the efficiency of iPSC induction through inhibition of p53/p21 pathway.

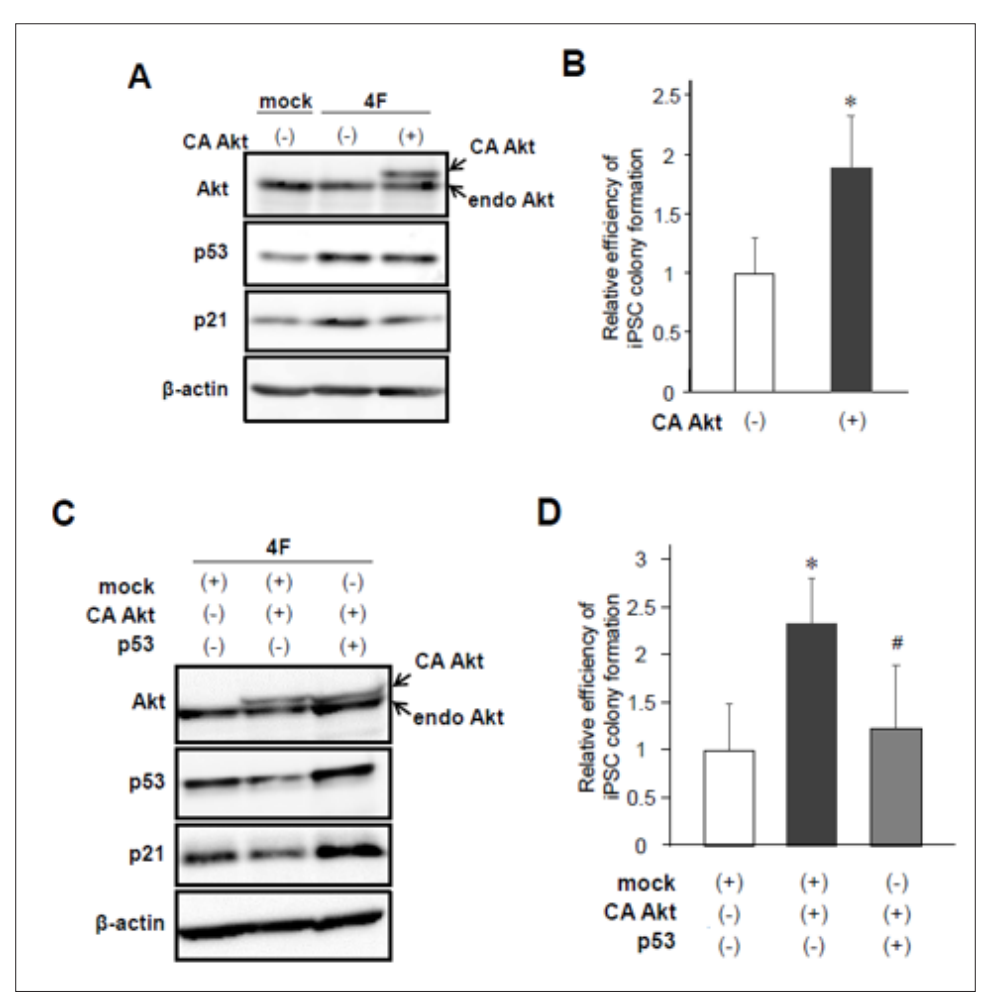

Figure 3: Effects of constitutively active Akt overexpression on iPSC reprogramming.

a) Protein levels of endogenous and constitutively active (CA) Akt, p53, and p21 after reprogramming induction.

b) Retroviral infection of CA Akt increased the number of Nanog-positive iPSC colonies. ( $n=7$, * $p<0.05$, error bars show s.d.).

c) Western blotting of Akt, p53, and p21 after reprogramming induction with or without co-expression of CA Akt and p53.

d) Forced expression of p53 canceled Akt-induced enhancement of iPSC colony formation ( $\mathrm{n}=6$, ${ }^{*} \mathrm{p}<0.05 \mathrm{vs}$ mock, \#p<0.05 vs CA Akt, error bars show s.d.).

\section{p 19Arf and p16Ink4a are also downregulated by Akt during reprogramming to iPSCs}

p19Arf, an upstream factor of p53, enhances p53 stabilization, whereasp16Ink4a, atranscriptvariantofp19Arf, p53-independently represses cell cycle. In addition, it was reported that silencing of p19Arf and p16Ink4a enhances iPSC reprogramming efficiency in part through a p53-dependent mechanism [6]. It remains unknown however, whether Akt is involved in p19Arf and p16Ink4adependent regulation of reprogramming. To examine the effect of
Akt activation on p19Arf and p16Ink4a expression, we performed western blotting using cell lysates after reprogramming induction with or without CA Akt. The protein levels of p19Arf and p16Ink4a were significantly decreased by co-infection of CA Akt (Figure 4A). Finally, we tested whether Akt-mediated downregulation of p19Arf and p16Ink4a is prevented by p53 activation. Overexpression of p53, however, did not affect this reduction. These data suggest that Akt improves the efficiency of reprogramming to iPSCs through p53-independent downregulation of p19Arf and p16Ink4a as well as directly affecting p53/p21 pathway (Figure 4B) 


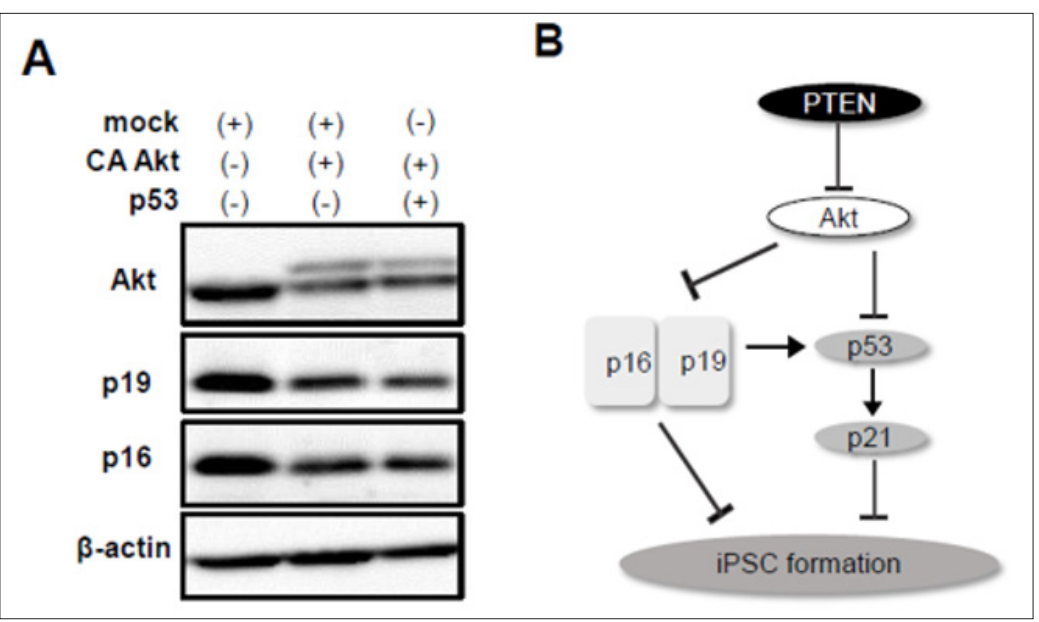

Figure 4: Involvement of $\mathrm{p} 19^{\text {Arf }}$ and $\mathrm{p} 16^{\text {Ink4a }}$ in reprogramming to iPSCs.

a) CA Akt reduced expression of p19 Arf and p16 ${ }^{\text {Ink4a }}$ which was not recovered by forced expression of p53.

b) Scheme of the signaling pathways of PTEN/Akt, p53/p21, and p19/p16 involved in iPSC formation.

\section{Discussion}

iPSCs have been massively studied to apply for regenerative medicine, disease modeling, or drug screenings. It is, however, still under investigation to dissect molecular mechanisms of iPSC reprogramming induced by the defined four factors, Oct4, Sox2, $\mathrm{Klf4}$, and c-Myc. We previously reported that p53 is activated after reprogramming induction and gene deletion or RNAimediated silencing of p53 remarkably increases the efficiency of reprogramming to mouse or human iPSCs [3]. Therefore, controlling the protein level or the stability of p53 by activation or inhibition of p53 regulators would be one of the strategies to establish iPSC lines efficiently. As a variety of cellular functions and signal transduction pathways associated with p53 have been widely investigated, a number of interacting molecules of p53 have been addressed so far. PTEN is a lipid phosphatase which directly or indirectly regulates the protein level and activity of p53 [20]. It was demonstrated that PTEN physically interacts with $\mathrm{p} 53$, contributes to cytosolic localization of p53, and prevents p53 protein from Mdm2-dependent degradation [21]. On the other hand, loss-offunction mutations in PTEN and p53, observed in patients with an aggressive brain tumor, are involved in the undifferentiated state of tumor progenitor cells with self-renewal potential, resulting in cancer progression [22]. Thus, we investigated, in the present study, whether inhibition of PTEN function is attributable to disruption of p53 stability that may result in efficient reprogramming to iPSCs. Our findings revealed that genetic ablation or CRISPR/Cas9mediated knock-down of PTEN promotes iPSC reprogramming efficiency through inhibition of p53/p21 pathway. We also showed correlated changes in protein levels among PTEN, phosphorylated Akt, p53, and p21, suggesting the crosstalk between PTEN/Akt and p53/p21 pathways involved in reprogramming to iPSCs.

In PTEN-deficient cells infected with 4F, Akt hyperphosphorylation and p53 downregulation were observed (Figure 1B). These findings suggest that Akt activation may facilitate p53 degradation regulated by E3 ubiquitin ligase, Mdm2 or Mdmx. Mdm2 is a well-known upstream protein that degrades p53 through ubiquitination, whereas Mdmx is the homologue of Mdm2 with similar enzyme activity. Our previous study showed that mutant Mdmx in which impaired phosphorylation in response to DNA damage or oncogenic stimuli enhanced reprogramming efficiency [3]. Findings above may support the evidence of contextdependent role of Mdmx observed in reprogrammed cells as well as various types of cancer cells [23]. Therefore, Mdm2 and Mdmx would be presumably involved in Akt-dependent p53 inactivation during iPSC reprogramming. Several studies revealed that PTEN regulates the protein level and activity of p53 through phosphataseindependent mechanisms [21]. p53 is able to bind to the promoter region of Pten that forms a positive feedback loop [24]. Although further studies are required to address underlying mechanisms to control Akt-independent p53 regulation by PTEN in iPSCs, PTEN downregulation appears to be one of the strategies to improve reprogramming efficiency. In addition, considering reprogramming $4 \mathrm{~F}$ activate the p53/p21 pathway, it might be interesting to investigate whether $4 \mathrm{~F}$ directly or indirectly regulate expressions or activation of PTEN and Akt in the course of iPSC formation.

Our present data also show that overexpression of CA Akt results in downregulation of p19Arf and p16Ink4a after reprogramming induction, nevertheless whether p53 is co-infected or not (Figure $4 \mathrm{~A})$. These results suggest that there might be p53-independent relationship between Akt and Ink4a-Arf locus in reprogrammed cells to iPSCs. We and other group previously reported that inhibition of p19Arf and p16Ink4a increased the number of iPSC colonies $[3,6]$. Therefore, enhanced reprogramming efficiency by Akt might be in part due to downregulation of p19Arf and p16Ink4a. As previously reported, Ink4a/Arf locus is transactivated during reprogramming, and $\mathrm{p} 19 \mathrm{Arf}$ and $\mathrm{p} 16$ Ink4a play a pivotal role in a critical barrier against reprogramming [6]. Therefore, activation of Akt or overexpression of its active mutant may contribute to silencing of Ink4a/Arf locus, leading to efficient iPSC generation. In conclusion, the present study revealed that genetic ablation or knock-down of PTEN improves the efficiency of reprogramming to 
iPSCs by activation of Akt at least in part through downregulation of p19Arf and p16Ink4a followed by p53 degradation as well as significant inhibition of $\mathrm{p} 53 / \mathrm{p} 21$ pathway (Figure 4B). Our findings suggest that modulating PTEN/Akt axis would be effective to enhance somatic cell reprogramming to pluripotency.

\section{Acknowledgement}

We are grateful to all members of the Laboratory of Stem Cell and Regenerative Medicine for helpful discussions. T.K. is a group leader of the research project supported by R-GIRO (Ritsumeikan Global Innovation Research Organization). T.S. is supported by R-GIRO. This work was supported by JSPS KAKENHI (T. K., S. N.), and by Takeda Science Foundation, Medical Research Grant (T. K.), TERUMO FOUNDATION for LIFE SCIENCES and ARTS, and NOVARTIS Foundation for the Promotion of Science (S. N.).

\section{References}

1. Takahashi K, Yamanaka S (2006) Induction of Pluripotent Stem Cells from Mouse Embryonic and Adult Fibroblast Cultures by Defined Factors. Cell 126(4): 663-676.

2. Takahashi K, Tanabe K, Ohnuki M, Narita M, Ichisaka T, et al. (2007) Induction of Pluripotent Stem Cells from Adult Human Fibroblasts by Defined Factors. Cell 131(5): 861-872.

3. Kawamura T, Suzuki, Wang YV, Menendez S, Morera LB, et al (2009) Linking the p53 tumour suppressor pathway to somatic cell reprogramming. Nature 460(7259): 1140-1145.

4. Hong H, Takahashi K, Ichisaka T, Aoi T, Kanagawa O, et al. (2009) Suppression of induced pluripotent stem cell generation by the p53-p21 pathway. Nature 460(7259): 1132-1135.

5. Marión RM, Strati K, Li H, Murga M, Blanco R, et al. (2009) A p53mediated DNA damage response limits reprogramming to ensure iPS cell genomic integrity. Nature 460(7259): 1149-1153.

6. Li H, Collado M, Villasante A, Strati K, Ortega S, et al. (2009) The Ink4/Arf locus is a barrier for iPS cell reprogramming. Nature 460(7259): 11361139.

7. Utikal J, Polo JM, Stadtfeld M, Maherali N, Kulalert W, et al. (2009) Immortalization eliminates a roadblock during cellular reprogramming into iPS cells. Nature 460(7259): 1145-1148.

8. Kastenhuber ER, Lowe SW (2017) Putting p53 in context. Cell 170(6): 1062-1078.

9. Wade M, Li Y, Wahl GM (2013) MDM2, MDMX and p53 in oncogenesis and cancer therapy. Nat Rev Cancer 13: 83-96.

\section{ISSN: 2574-1241}

DOI: $10.26717 / B J S T R .2018 .11 .002162$

Tomoe Ueyama. Biomed J Sci \& Tech Res

This work is licensed under Creative

Commons Attribution 4.0 License

Submission Link: https://biomedres.us/submit-manuscript.php
10. Green DR, Koremer G (2009) Cytoplasmic functions of the tumour suppressor p53. Nature 458(7242): 1127-1130.

11. Wang YV, Leblanc M, Wade M, Jochemsen AG, Wahl GM (2009) Increased radioresistance and accelerated $\mathrm{B}$ cell lymphomas in mice with Mdmx mutations that prevent modifications by DNA-damage-activated kinases. Cancer Cell 16(1): 33-43.

12. Liang J, Slingerland JM (2003) Multiple roles of the PI3K/PKB (Akt) pathway in cell cycle progression. Cell Cycle 2(4): 339-345.

13. Haupt Y, Maya R, Kazaz A, Oren M (1997) Mdm2 promotes the rapid degradation of p53. Nature 387(6630): 296-299.

14. Kubbutat MH, Jones, SN, Vousden KH (1997) Regulation of p53 stability by Mdm2. Nature 387(6630): 299-303.

15. Honda R, Tanaka H, Yasuda H (1997) Oncoprotein MDM2 is a ubiquitin ligase E3 for tumor suppressor p53. FEBS 420(1): 25-27.

16. Mayo LD and Donner DB (2001) A phosphatidylinositol 3-kinase/Akt pathway promotes translocation of Mdm2 from the cytoplasm to the nucleus. Proc Natl Acad Sci USA 98(20): 11598-11603.

17. Stambolic V, Suzuki A, de la Pompa JL, Brothers GM, Mirtsos C, et al. (1998) Negative Regulation of PKB/Akt- Dependent Cell Survival by the Tumor Suppressor PTEN. Cell 95(1): 29-39.

18. Ahmed NN, Grimes HL, Bellacosa A, Chan TO, Tsichlis PN (1997) Transduction of interleukin-2 antiapoptotic and proliferative signals via Akt protein kinase. Proc Natl Acad Sci U S A 94(8): 3627-3632.

19. Ran FA, Hsu PD, Wright J, Agarwala V, Scott DA, et al. (2013) Genome engineering using the CRISPR-Cas9 system. Nat Protoc 8(11): 22812308.

20. Freeman DJ, Li AG, Wei G, Li HH, Kertesz N, et al. (2003) PTEN tumor suppressor regulates p53 protein levels and activity through phosphatase-dependent and -independent mechanisms. Cancer Cell 3: $117-130$

21. Zhou M, Gu L, Findley HW, Jiang R, Woods WG (2003) PTEN reverses MDM2-mediated chemotherapy resistance by interacting with p53 in acute lymphoblastic leukemia cells. Cancer Res 63(19): 6357-6362.

22. Zheng H, Ying H, Yan H, Kimmelman AC, Hiller DJ, et al. (2008) p53 and Pten control neural and glioma stem/progenitor cell renewal and differentiation. Nature 455(7216): 1129-1133.

23. Marine JC, Dyer MA, Jochemsen AG (2007) MDMX: from bench to bedside. J Cell Sci 120(3): 371-378.

24. Stambolic V, MacPherson D, Sas D, Lin Y, Snow B, et al. (2001) Regulation of PTEN transcription by p53. Mol Cell 8(2): 317-325.

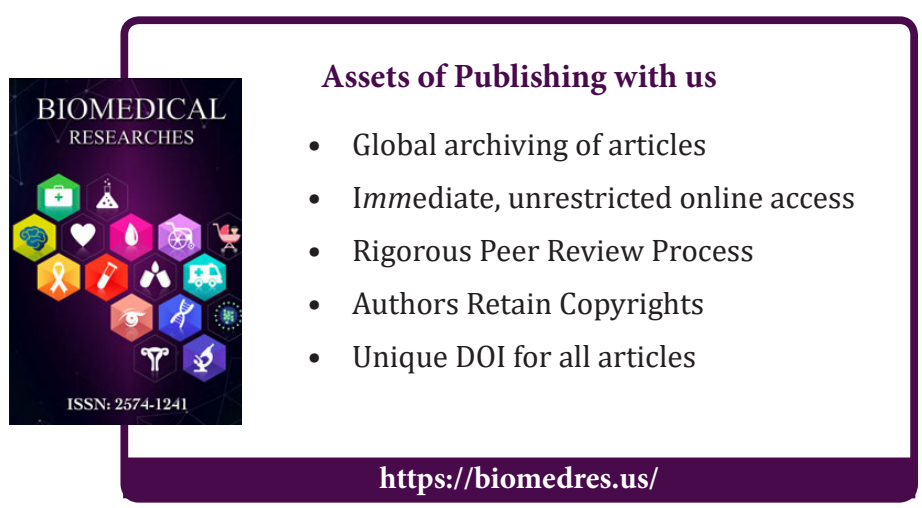

